\title{
El Torno, balance socioeconómico de un minicipio del valle del Jerte
}

\author{
Ricardo Salaya
}

\begin{abstract}
RESUMEN
ABSTRACT

El estudio de El Torno, situado en el valle del Jerte, pone de relieve los recursos naturales y económicos que pueden ofrecer oportunidades para su desarrollo y que podrian paliar su actual

\author{
The study of El Torno, located in the \\ Jerte valley, emphasizes its natural \\ and economic resources than can \\ possible avoided its situation of \\ demographic decline.
} situación de agotamiento demográfico.

\section{EL MEDIO NATURAL}

El Valle del Jerte está constituido por una amplia falla diagonal de dirección ENE-OSO, que se inscribe en el conjunto estructural de la Cordillera Central, separando las Sierras de Gredos, al Sur, y Béjar, al Norte, y está conformado por materiales graníticos.

En su extremo noroccidental, en la vertiente sur de los Montes de Tras la Sierra, se localiza El Torno, cuyo relieve se articula alrededor de la gran falla central del valle, y cuatro fallas menores que definen una serie de zonas de pendiente y rellanos estructurales que condicionarán los usos del suelo.

Su orientación ENE-OSO es determinante de su principal característica climática: la abundancia de precipitaciones, al quedar abierto a la penetración de los vientos ábregos durante la primavera y el otoño. 
Frente a ellos, el valle se comporta como un embudo, y dirige su ascensión desde los $350 \mathrm{~m}$. de altitud en Plasencia, hasta los 1275 del Puerto de Tornavacas, salvando un desnivel de $925 \mathrm{~m}$. en apenas $50 \mathrm{~km}$., lo que provoca un incremento de las precipitaciones con respecto a las áreas circundantes, que se intensifica a medida que gana altitud ${ }^{1}$.

Las temperaturas son suaves, como corresponde a un clima húmedo, con medias anuales alrededor de los $13^{\circ} \mathrm{C}$. La media de las máximas se sitúa en los $19^{\circ} \mathrm{C}$ y la de las mínimas en los $7,5^{\circ} \mathrm{C}$.

La aridez estival se concentra en los meses de julio y agosto (considerando las medias), aunque esporádicamente puede aparecer entre junio y septiembre.

Con estos datos, podemos clasificar el clima en El Torno como mediterráneo de montaña, o mediterráneo húmedo, aunque se podrían introducir matizaciones y llegar a una caracterización agroclimática dividiendo el término municipal entre mediterráneo subtropical y mediterráneo templado ${ }^{2}$ según la clasificación de Papadakis que, como veremos, se corresponden perfectamente con los modos de aprovechamiento tradicional.

Entre las formaciones vegetales podemos diferenciar las que han sido más alteradas: praderas en las que se controla la penetración del matorral; antiguas formaciones boscosas aclaradas, que actualmente se explotan como monte hueco, y tierras abancaladas para el cultivo de frutales, en las que la transformación resulta radical.

Por lo que respecta a aquellas en las que la intervención humana ha sido menos intensa, ha cesado o ha introducido modificaciones menos aparatosas y presentan una apariencia más natural, se diferencian unidades con predominio del matorral, fundamentalmente de ericaceas, Cytisus, Echinospartun, etc. y unidades con predominio de la cubierta arbórea, entre las que destacan especialmente los rebollares (Quercus pyrenaica). Mención aparte merecen dos formaciones presentes en la zona: la primera son los roquedos, que aparecen distribuidos en todo el término municipal, aunque con mayor presencia en las zonas de mayor altitud y pendiente, formando manchas de superficie considerable en las que la colonización vegetal supondrá un trabajo de siglos y la única cobertura actual está constituida por musgos y líquenes 0 , en el mejor de los casos, por un desarrollo subarbustivo intersticial a base de Lavándula ssp., Thi-

\footnotetext{
TORROJA habla de una precipitación media anual cercana a los $2000 \mathrm{~mm}$., en las zonas más altás de la sierra de Tormantos, aunque no existen observatorios en esas altitudes.

2 TORROJA, J. A.
} 
mus ssp., y alguna vegetación herbácea de gramíneas. La segunda es la que corresponde a la vegetación ripícola, que presenta, en buena parte de la margen del río Jerte y de las diferentes gargantas, unas condiciones de conservación excelentes, constituyendo formaciones de gran riqueza florística, en las que los estratos arbustivo y arbóreo alcanzan una cabida cubierta ${ }^{3}$ cercana al $100 \%$.

La intervención secular del hombre sobre el medio, que alcanza su máxima manifestación en las gavias (bancales soportados por paredes de mampostería seca), se ha producido con gran intensidad, pero generando en la mayor parte de los casos formas muy integradas en el paisaje.

La diversidad florística, junto con lo quebrado del terreno, da lugar a un mosaico complejo, en el que se produce una perfecta integración de las estructuras productivas en un medio que, sin poder ser considerado natural - de acuerdo a los criterios biogeográficos al uso- si responde al concepto de naturaleza generalizado en nuestra sociedad, convirtiéndose en un recurso explotable.

\section{POBLACIÓN: UNA SANGRIA DEMOGRÁFICA SOSTENIDA}

La evolución de la población a lo largo del último siglo muestra claramente los caracteres comunes al conjunto del medio rural en España: notable crecimiento hasta los años 50 , momento de inflexión a partir del cual se produce una caída sostenida que continúa hasta nuestros días, leve recuperación durante los 80 , insuficiente para paliar los efectos del éxodo, y vuelta en los 90 a la recesión -esta vez como consecuencia de la estructura demográfica - sin que se adivine el fin del proceso.

La población se encuentra por debajo del nivel de principios de siglo, aspecto en que coincide con Casas del Castañar y Tornavacas, constituyendo el grupo más regresivo de una comarca en la que la regresión demográfica es la norma, a excepción de Cabrero, Cabezuela del Valle y, sobre todo, Navaconcejo, que durante este último siglo se beneficia de su posición central.

Por lo que respecta a su participación en la comarca: el decrecimiento es constante desde 1900, y apenas se percibe un mínimo repunte en la segunda mitad de los 80 y primera de los 90 ; pese a ello, ha pasado

3 Cabida cubierta: porcentaje de la superficie del suelo que ocuparia la proyección de las copas de los árboles o arbustos. 
desde un $11,25 \%$ a un $7,98 \%$ en 1998 . Este fenómeno es común a la totalidad de los pueblos, a excepción de Navaconcejo y Cabezuela del Valle: en los momentos en que la comarca muestra cierta recuperación, ésta se concentra en dos pueblos. Por tanto, nos encontramos con un ámbito en el que el fenómeno del éxodo rural provoca una regresión demográfica a partir de 1950, aunque suavizada, de modo que el número de habitantes en 1998 es superior al de principios del siglo, pese a lo cual probablemente nunca llegue a alcanzar el de 1950. Este dato encubre grandes diferencias internas: la mayor parte de la comarca manifiesta una pérdida neta de población, mientras que dos pueblos ${ }^{4}$, Cabezuela y Navaconcejo, polarizan la totalidad del crecimiento demográfico comarcal.

Esta evolución se manifiesta en la estructura demográfica: los grupos susceptibles de emigrar son los más dinámicos y en ellos se integran los elementos reproductores, de modo que, tras un movimiento migratorio como el padecido, la población se va a ver afectada por un proceso de envejecimiento progresivo que tiende a retroalimentarse, dado que las condiciones subjetivas de vida en el pueblo se hacen peores a medida que aumenta la edad media de sus habitantes. Este fenómeno podría verse suavizado por la mejora en el nivel de ingresos derivada de la posibilidad de incrementar el tamaño de la explotación con las tierras de los que se marchan, pero en el caso de El Torno, la escasa entidad del terrazgo no permite, ni aun así, una ampliación suficiente.

Como consecuencia, nos vamos a encontrar con una estructura por edades muy envejecida (Índice de envejecimiento $=2,6$ ), especialmente comparándola con su equivalente en Navaconcejo ${ }^{5}$, y muy desequilibrada en cuanto a sexos: la sex ratio es 1,06 para el conjunto, pero asciende a 1,16 si nos limitamos a la población de 0 a 64 años, y muestra una estructura aún peor en el grupo de edades de 15 a 39 años $(1,24)$, que es donde debería concentrarse la mayor parte de los nacimientos ${ }^{6}$.

Se trata de una situación común al medio rural en el interior de España, y El Torno muestra los caracteres del conjunto del Valle muy acentuados, puesto que estos se ven fuertemente alterados por la influencia de Nava-

4 También lo hacen Jerte y El Cabrero, aunque en términos absolutos su crecimiento carece de importancia.

5 Hemos utilizado el \% de cada grupo de edad referido al total de población de cada género para asi homogeneizar ambas pirámides. La agrupación en 4 clases trata de evitar la excesiva incidencia de cada "accidente" demográfico cuando se analizan poblaciones de tan escasa entidad.

6 Este fenómeno aparece muy frecuentemente asociado a áreas afectadas por el éxodo rural en las que las condiciones de la reproducción del grupo hacen pensar en una decadencia dificilmente reversible. 
concejo y Cabezuela del Valle que, al ofrecer mayores resistencias a la despoblación, consiguen mantener una estructura más joven y equilibrada, aunque no pueda afirmarse tampoco que se encuentren en una situación en la que tengan asegurada una dinámica positiva.

Una estructura como la que acabamos de ver condiciona fuertemente el movimiento natural de la población, y sus resultados se aprecian tanto en el balance demográfico, como en el conjunto de los indicadores asociados a la capacidad reproductora del grupo.

Se puede afirmar que el proceso de despoblación inducido a partir de los años 50 y sostenido de manera espontánea más tarde, tiene la capacidad de retroalimentarse, no sólo por la pérdida de población que implica per se, sino también por lo que significa: por el ambiente de decadencia, que motiva la apatía reproductora; por falta de incentivos para afrontar la puesta en marcha de una nueva explotación etc.

Así, nos encontramos con una tasa bruta de natalidad de apenas 5,5 0/00 (9,7 0,00 para Extremadura), enfrentada a una tasa bruta de mortalidad de 8,2 0/00 (7,4 0/00 para Extremadura), que se traducen en un crecimiento vegetativo de $-0,270 / 0^{7}(0,050 / 0 \text { para Extremadura })^{8}$. Una pérdida media para el periodo 1991-1999 del 0,27\% anual de su población, agravada si sólo tuviésemos en cuenta el periodo de 1996 a 1999, pues la tasa de natalidad quedaría reducida al 4,5 o/00, mientras que la de mortalidad ascendería hasta el 9,8 0/00, arrojando un crecimiento vegetativo $-0,5300$.

$Y$ es que con una tasa de fecundidad efectiva de apenas el 20,69 0/00, sumada a la deficiente estructura por edades, las posibilidades de supervivencia de El Torno ni siquiera están garantizadas a medio plazo, pudiendo considerarse que tomando en cuenta sólo las inercias a que hacíamos referencia, se encuentra condenado a la despoblación.

Por lo que respecta a la población analizada en términos de capital humano (mercado laboral y niveles formativos), la situación no es más halagüeña:

\footnotetext{
Utilizamos la media de los datos de 1991 a 1999 a fin de evitar la distorsión que cada caso concreto puede introducir en poblaciones tan pequeñas. Fuente: Registro Civil de El Torno.

8 Datos de TBM, TBM y CV referidos a la región correspondientes al año 1996. Fuente: Junta de Extremadura: "Anuario estadístico de Extremadura, 1999", que cita INE, Movimiento Natural de la Población Española.
} 
Nivel de estudios en la población de El Torno

\begin{tabular}{lrr}
\hline \multicolumn{1}{c}{ Nivel de estudios } & N. & $\%$ \\
\hline No sabe leer ni escribir & 26 & 2,66 \\
Sin estudios & 393 & 40,27 \\
Primaria incompleta & 112 & 11,48 \\
Graduado escolar o similar & 347 & 35,56 \\
FP1 & 4 & 0,41 \\
FP2 & 14 & 1,43 \\
Bachiller superior & 44 & 4,51 \\
Diplomados universitarios y técnicos & & \\
de grado medio & 16 & 1,64 \\
Licenciados universitarios & 20 & 2,05 \\
\hline
\end{tabular}

Elaboración propia a partir de Ayuntamiento de El Torno. Actualización permanente del Padrón Municipal de Habitantes (consulta a 3-7-2000).

Lo primero que nos encontramos al analizar las condiciones de la población activa es que, como consecuencia de la estructura y la dinámica demográfica reciente y actual, existe un índice de reemplazo de la población activa que apenas alcanza 0,64 , y una relación de dependencia de 0,68 .

Ambos indicadores son significativos por cuanto que llevan a pensar en un decrecimiento irreversible de la capacidad de la población para asegurar el sostenimiento de las bases productivas actuales.

La previsible disminución de la superficie agrícola en explotación, o la sustitución de sus titulares por otros "forasteros", supone un paso cualitativo más en la consolidación de ese ambiente de decadencia al que ya se ha hecho mención, y que tan negativamente incide en las posibilidades de recuperación.

Las cifras relativas a los niveles formativos son también muy ilustrativas: en los primeros 5 grupos (graduado escolar o inferior) encontramos a un $89,97 \%$ de la población total, cifra esta que basta cotejar con el porcentaje que supone la población menor de 30 años $(27,87 \%)$ para percibir la existencia de un notable fenómeno de abandono de los estudios en niveles iguales o inferiores a la educación básica, o del abandono del pueblo por un porcentaje significativo de los que completan estudios medios y superiores. Más ilustrativo si cabe es el porcentaje de población que ha terminado al menos la enseñanza secundaria superior, que supone, en el caso que nos ocupa, apenas un $9,6 \%$, frente al $36,0 \%$ de media nacional (ya de por sí baja frente al $59,9 \%$ de media en la Unión Europea, e incomparable con las cifras superiores al $70 \%$ en 5 de los países miem- 
bros) ${ }^{9}$. Se trata de un fenómeno grave, por cuanto supone un derroche de capital humano potencial, en el primero de ellos, o un drenaje de recursos en el segundo, que se constituye en un factor más de decadencia.

El análisis del mercado laboral resulta dificultoso por la ausencia de fuentes de información fiables provocada por la perturbación que introduce el AEPSA (antiguo PER), que genera formas de ocultación de los ingresos encaminadas a mantener la percepción del subsidio de desempleo agrario, y dificulta la diferenciación entre parados nominales y reales.

Baste, para hacerse una idea de la magnitud del fenómeno, considerar la presencia en el pueblo de 78 perceptores de esta prestación, el $13,45 \%$ de la población potencialmente activa, cifra claramente exagerada. Un rosario de prácticas acerca de las que tendremos ocasión de tratar más adelante hace inútiles todas las fuentes que tratan de registrar el paso en el conjunto del medio rural extremeño, y muy particularmente en zonas menos desfavorecidas, como es el caso del Valle del Jerte. Más próxima a la realidad se encuentra la idea de una situación cercana al pleno empleo en el Valle del Jerte en su conjunto, de la que no es ajeno El Torno (sin obviar la existencia de diferencias sustanciales en los niveles de ingresos) en la que, la necesidad de declarar rentas inferiores a un determinado umbral, lleva a la búsqueda de fórmulas legales de ocultación y al florecimiento de prácticas informales en el mercado laboral.

Una población muy envejecida, decreciente desde hace décadas, y que no muestra ningún síntoma de ir a cesar en esta tendencia, sitúa a $\mathrm{El}$ Torno, si es que no ha llegado ya, a punto del colapso de su capacidad de reproducción.

Esto se produce en un contexto de desánimo, de falta de confianza en las propias potencialidades, convirtiéndolo en un espacio muy poco atractivo para la captación de población foránea, o para albergar la esperanza de que se vaya a producir un movimiento de retorno de dimensiones suficientes para paliar el problema, ni siquiera en el caso de una recesión económica, que hiciera más atractivos los espacios rurales de origen a los desempleados inmigrantes en medio urbano.

En estas circunstancias, las posibilidades de revitalización son escasas por la propia dinámica local, y la única esperanza de hacer revertir el proceso tiene que basarse en la adopción de medidas decididas de dinami-

9 Fuente: "España en cifras" Instituto Nacional. (WEB) consulta a 28-3-2001. En http: Ilwww.ine.es. 
zación que lleven a la aparición de iniciativas capaces de alterar la inercia, dado que las condiciones económicas, como podremos comprobar, no justifican la situación de regresión en que se encuentra.

\section{EL PROCESO DE DESPOBLACIÓN Y SU TRADUCCIÓN EN LAS ESTRUCTURAS ECONÓMICAS Y TERRITORIALES}

La década de los años 50 en España es la del despegue industrial, que se produce de manera tardia, y sólo gracias a una decidida acción institucional que propiciará el trasvase masivo de recursos desde el medio rural agrario hacia las áreas industriales emergentes.

Este trasvase, cuyo detalle no vamos a entrar a analizar aquí, se produce fundamentalmente a través de dos mecanismos:

- Fomento de movimientos masivos de población rural hacia áreas industriales.

- Mantenimiento de precios bajos en los productos alimenticios, que permitan asegurar la reproducción de la fuerza laboral para la industria en condiciones de salarios competitivos.

El medio rural, como consecuencia de las mejoras que se producen durante la primera mitad del siglo $x x$ en las condiciones de la lucha contra la muerte, conoce hasta 1950 una expansión demográfica sin precedentes, que se traduce en un notable empeoramiento de las condiciones laborales. El terrazgo apenas puede crecer ya sin avanzar hacia tierras de escasa calidad agronómica, y la estructura desequilibrada de la propiedad de los factores de producción dificulta el logro de niveles de vida aceptables.

En este contexto surge la posibilidad de desplazamiento hacia las áreas urbanas, muy atractiva para grandes sectores de la población rural -especialmente jóvenes con dificultades para incorporarse a la explotación agraria- que a partir de los 50 comienzan en un goteo sostenido a emigrar hacia ellas.

Las importantes diferencias entre los niveles salariales y la existencia de condiciones regladas de trabajo, no tardan en percibirse a través de las visitas de los primeros emigrantes, cuyos relatos ejercen un poderoso efecto de llamada, que se une a los factores expulsores que ya se venían produciendo, acelerándose el proceso durante la década de los 60 , e incluso manteniéndose, aunque atenuado, en la primera mitad de $\operatorname{los} 70$. 
Este movimiento, que en su inicio sólo había afectado a población excedentaria, al generalizarse producirá disfuncionalidades en la explotación agraria que, unidas a las demandas de mayor productividad, a fin de mantener los precios de alimentos y materias primas para el desarrollo de la industria, lleva a profundizar en la mecanización en lo que podríamos considerar una auténtica revolución verde, que a su vez genera nuevos excedentes de población, interactuando de este modo ambos factores de la dinámica expulsora.

Simultáneamente, se produce una mejora en los modos de transporte, que permite modificar las condiciones de subsistencia de la agricultura tradicional y buscar nuevas orientaciones productivas con destino a los mercados urbanos.

Este proceso, cuyo desencadenamiento viene dado por voluntad política, se escapa de las manos de la administración, generando grandes disfuncionalidades en la explotación agraria y poniendo en cuestión la capacidad productiva de nuestra agricultura, lo que obliga, a su vez, al planteamiento de políticas de desarrollo rural y reforma de estructuras que tratan de paliar sus efectos perversos.

Ya hemos podido ver los efectos demográficos que el fenómeno del éxodo rural desencadena en la localidad objeto de este estudio, pero nos falta constatar su incidencia sobre las estructuras territoriales, que van a producir una alteración radical tanto de las orientaciones productivas, como de los modos de explotación.

A principios de los años 50 , la economía jerteña se basa fundamentalmente en el cultivo del olivo, la vid, el castaño, el cerezo - subordinado a los anteriores - y una producción de subsistencia en la que encontramos desde naranjos hasta patatas. Junto a todo ello, existe una ganadería relativamente próspera en régimen de trashumancia, que explota los grandes llanos serranos.

La pérdida de población, la mejora en los medios de transporte, y la decadencia del castaño provocada por la «tinta» ${ }^{10}$, van a ocasionar una reorientación productiva, que se vierte cada vez más hacia el cerezo, producto de primor que alcanza elevados precios y permite, ante la reducción de la mano de obra disponible, la supervivencia mediante la gestión de pequeñas explotaciones.

10 Micosis producida por Phytophtora cambivora y Ph. Cinnamomi que causa la muerte de varias especies arboreas (fundamentalmente Castanea ssp) que hace su aparición en la comarca a mediados del siglo xx (Fuente: CEVALLOS y FERNÁNDEZ DE CÓRDOBA, L. et. alter, 1979). 
Hasta entonces, las dificultades del traslado hacia los mercados urbanos de un producto extremadamente delicado, que no permite viajes prolongados a temperatura ambiente, había limitado su expansión. Sin embargo, las mejoras en la infraestructura de transporte y del parque de vehículos, incrementan el área de mercado, que se extiende ya en los años 70 a todo el territorio nacional.

Por primera vez, el Valle se especializa en un producto valioso para el que se encuentra especialmente dotado y en el que existe una tradición previa, que permite su generalización sin más dificultades técnicas que las derivadas de lo infractuoso del terreno, ya resueltas mediante la titánica tarea de construcción del sistema de gavias que convierten a su paisaje en excepcional.

Esta especialización implica la decadencia del resto de los cultivos, que se manifiesta en menor medida en El Torno que en el resto del valle, con una pervivencia del castaño y el olivo que permite, aún en nuestros días, producciones de cierta importancia económica.

Sin embargo, lo breve del periodo de cosecha, unido a la imposibilidad de mantener la fruta en el árbol durante un tiempo en condiciones de ser cosechada, genera una gran necesidad de mano de obra concentrada en apenas tres meses al año, que dificulta la formación de grandes unidades de explotación en las condiciones generadas por el éxodo. Este problema se solventará en un principio mediante la captación de mano de obra temporal en áreas menos favorecidas (fundamentalmente las Hurdes), pero la mejora en las condiciones de vida alcanza también a esta comarca, especialmente a partir de los años 80 , y no tardará en reducir la disponibilidad de trabajadores.

Esta escasez de mano de obra asalariada va a ser la principal limitación, junto con las dificultades introducidas por criterios ambientalistas, a la roturación de nuevas tierras para el aumento del tamaño de la explotación, y va a ser causa de un cambio en las técnicas de cultivo que, especialmente a lo largo de la década de los 90 , se traducirá en una alteración fisonómica notable, desapareciendo prácticamente en un periodo de 10 ó 12 años los enormes cerezos que dieron al Valle su aspecto peculiar, y alterándose el balance de color del paisaje hacia los ocres durante el periodo estival.

A lo largo de este proceso, la ganadería, la explotación maderera, el cultivo de la vid, la producción hortícola etc. van a ir marginalizándose, hasta adquirir el carácter de vestigios de otros tiempos, o mantenerse apenas como complementos subsistenciales a la economía familiar. 
En apenas 50 años, hemos podido asistir a la transformación de una tierra pobre de montaña en una comarca floreciente, especializada en un producto valioso, que no deja de extender sus áreas de mercado. Sin embargo, no ha sido suficiente para evitar la despoblación, el colapso demográfico de buena parte de sus pueblos, que, paradójicamente, asisten a su vaciamiento en el momento en que mayores son sus potencialidades y mejores las condiciones de vida.

Las características de la explotación resultan muy ilustrativas acerca de la capacidad de adaptación de las gentes del lugar a las cambiantes condiciones del medio demográfico y económico.

El factor que caracteriza a la empresa agraria en el valle del Jerte es la escasez de tierras ${ }^{11}$ y la dispersión en parcelas de cada una de ellas. La primera supone un problema de difícil solución, y respecto a la segunda cabe hacer la matización de que en un cultivo en el que las tareas de recolección afectan cada día a un número muy reducido de pies por explotación, supone un problema de escasa entidad si la selección de variedades por parcela es adecuada, pues no supone desplazamientos innecesarios a lo largo de la época durante la cual se concentra el grueso del trabajo.

Al buscar información respecto a sus dimensiones, que nos permita caracterizar lo que podríamos denominar una explotación-tipo, nos encontramos con el problema que supone la escasa validez de los datos disponibles, afectados por la inclusión a efectos fiscales de terrenos que en la actualidad resultan económicamente improductivos (pastos de montaña con frecuencia invadidos por vegetación leñosa). Si tuviéramos en cuenta la superficie agraria teórica, por tanto, dispondríamos de una explotación media cuya superficie estaría cerca de las $11 \mathrm{Ha}$, extremo éste que, desde luego, no se ajusta a la realidad. Por otra parte, la excesiva parcelación de la propiedad y la escasa extensión de los términos municipales en la comarca, hace que una parte de la superficie explotada por un titular se encuentre con frecuencia en un término municipal diferente, dificultando la utilización del catastro de rústica.

Los socios de la cooperativa ${ }^{12}$ son en la actualidad 647 , aunque hay que considerar que es práctica habitual que aparezcan como tales varios miembros de la unidad familiar, y que está generalizada la aparcería, con

\footnotetext{
"Las tierras dedicadas al cultivo en el Valle del Jerte apenas suponen un ratio de 0,55 Ha/persona, pero se reducen en El Torno hasta 0,21

12 El Torno se caracteriza por tener uno de los porcentajes de pertenencia a la cooperativa más importante del Valle del Jerte, acercándose mucho al $100 \%$.
} 
lo que en realidad una única explotación puede encontrarse registrada a nombre de hasta tres y cuatro titulares diferentes en el Catastro.

Para aproximarnos al número de explotaciones con un menor margen de error, podemos utilizar el listado del impuesto sobre vehículos de tracción mecánica (IVTM), contando los vehículos del tipo que se utilizan únicamente para la explotación agraria en la comarca (vehículos todo-terreno y furgonetas "combi» tipo Renault express, Citroën $\mathrm{C} 15$, etc.). Su número, 19 en el primer caso y 200 en el segundo, sí arroja unas cifras razonables en torno a la cantidad de explotaciones agrícolas funcionales ${ }^{13}$. De este modo, y excluyendo un $10 \%$ de la superficie agraria útil (SAU), correspondiente a explotaciones cuyas dimensiones son insuficientes para el desarrollo a título principal de la explotación agraria, que eliminaremos del cómputo, y dando por buena la cifra de 219 explotaciones funcionales, la superficie media por explotación se situaría en 1,27 Ha ${ }^{14}$.

La aproximación a sus características nos la puede dar el análisis de producciones comercializadas a través de la cooperativa agraria San Lucas:

Tenemos que considerar que las producciones que se encuentran repartidas por todas las explotaciones son las de cerezas, aceitunas, y castañas, mientras que frambuesas y ciruelas están muy concentradas (apenas 21 socios producen la totalidad de la frambuesa en 1999) ${ }^{15}$. Por ello, para establecer una caracterización de la explotación prescindiremos de ellas, sin que se resienta en exceso nuestro cálculo.

Tenemos, por consiguiente, una explotación agraria de escasa dimensión media $(1,27 \mathrm{Ha})$, cuya producción en términos monetarios asciende a tan solo 1.168.907 pts./año, y que, sin embargo, mantiene a sus propietarios en condiciones económicas más que aceptables, lo cual parece de todo punto imposible.

La explicación hay que buscarla en múltiples factores: por una parte, la explotación produce, al tiempo que los productos comercializables, buena

13 Naturalmente, seguimos sujetos a error, pero este será menor que usando cualquiera de las demás fuentes citadas, y lamentablemente no hemos tenido la oportunidad de corsultar el Registro de Explotaciones, cuyo acceso se ha visto muy dificultado desde la desaparición de las Cámaras Agrarias.

14 Excluimos todas las superficies no dedicadas directamente a la producción de frutas, cuyo rendimiento no pasa de simbólico.

15 Elaboración propia a partir de datos proporcionados por Sociedad Cooperativa "San Lucas". 
parte de los alimentos consumidos por la unidad familiar ${ }^{16}$ (frutas, verduras, carnes ${ }^{17}$, huevos etc.); por otra, como resultado de la estructura por edades de la población, una buena parte de los habitantes ${ }^{18}$ de El Torno son perceptores de pensiones de jubilación, y un total de 78 de ellos perciben a su vez el subsidio de desempleo agrario, que conlleva además el trabajo por cuenta ajena durante una cantidad variable de jornadas al año, que oscilan entre ninguna (mayores de 55 años) y 60 . Todo ello, junto con una explotación ganadera ya decadente, pero que ocupa a algunos de los vecinos todavía; la actividad terciaria, y el trabajo en la construcción ${ }^{19}$, complementa un panorama de ingresos basado en la pluriactividad, que, sin excesos, permite el mantenimiento de un nivel de vida aceptable, en una localidad donde la vivienda en alquiler es una excepción.

Por lo que respecta al grado de parcelación, cuyo significado ya ha sido mencionado a lo largo del estudio, es extremo, contabilizándose en el pueblo un total de 3116 parcelas ${ }^{20}$.

En definitiva, se trata de una explotación claramente disfuncional, entre cuyas carencias destaca claramente la escasez de superficie. Ésta, que se solventará en breve plazo - basta para comprobarlo examinar los indicadores de reemplazo generacional- probablemente no es tan aparatosa como las cifras parecen indicar, y para comprobarlo habría que indagar también en los catastros de Valdastillas y Rebollar, en cuyos términos municipales se encuentra una parte de la superficie explotada por los agricultores torniegos. En cualquier caso, a medio y largo plazo la preocupación debe centrarse más en la falta de brazos para trabajarla que en las dimensiones actuales, pues éste parece ir a ser el problema que se manifieste con mayor intensidad en el plazo de 10 a 12 años, cuando se haya retirado del mercado un $25 \%$ de la población potencialmente activa actual, sin que existan efectivos para reemplazarla.

16 Su estimación en términos monetarios supondria en torno a las 500.000 ptas /año (Fuente: Rosa JuLián).

17 El grueso de las carnes consumidas en la comarca proceden de la matanza, práctica tradicional que se mantiene vigente de un modo generalizado.

18 Hay que considerar, junto con las pensiones contributivas, que cubren a la práctica totalidad de los mayores de 65 años, un total de 44 perceptores de prestaciones diversas: FAS, PNC por invalidez o jubilación. LISMI... (Fuente: Estrella DE MIGUEL, del SSB).

19 Cuya cuantificación vuelve a ser difícil a causa del ya mencionado problema de la economía intormal en el medio rural a escala regional.

20 Naturalmente, ésta es una descripción estándar, en cuyo interior se ocultan enormes disparidades, que van desde, por ejemplo, la existencia de una explotación cuya superficie es de 21,7 Ha dedicadas al cultivo, que se encuentra repartida en tan sólo 27 parcelas, frente a otras que totalizan hasta 20 parcelas para una superficie total de $3,6 \mathrm{Ha}$. 
En cuanto al progreso técnico, el grado de mecanización sigue siendo bajo, debido más a las características del terrazgo que a otros factores. De hecho, la incorporación al mercado de pequeños tractores agrícolas, desbrozadoras manuales, fumigadoras, etc. va poco a poco terminando con el empleo de animales de tiro y facilitando determinadas labores especialmente penosas. El consumo de insumos es elevado, en parte por las dificultades para la mecanización, que están llevando a algunos agricultores a la práctica de formas de agricultura sin labor, basadas en el empleo de herbicidas (aunque no existe consenso acerca de su conveniencia, y la mayor parte de la tierra se labra por lo menos dos veces en el curso del año). Tampoco es escaso el consumo de abonos y pesticidas, cada vez más necesarios estos últimos por el afianzamiento del fenómeno plaga, inevitable en un área en monocultivo.

\section{PAISAJE Y POBLAMIENTO}

No cabe duda de que el paisaje constituye un recurso económico de primer orden, cuya puesta en valor depende, entre otros factores, de su espectacularidad, condicionada a su vez por las condiciones de conservación y la accesibilidad desde las aglomeraciones.

A este respecto, el Valle del Jerte goza de un recurso de extraordinario valor, especialmente si consideramos que las ya excepcionales cualidades del medio natural, se ven complementadas aquí por una intervención humana, que incrementa su espectacularidad, y por la especialización en el cultivo del cerezo, que sobre todo durante la primavera temprana, con la floración, y el otoño, cuando la gran variedad de especies arbóreas le dota de una extraordinaria gama de rojos y dorados, lo convierten en uno de los lugares más atractivos accesibles desde Madrid en menos de 3 horas.

La belleza de sus paisajes, la bonanza del clima, la excepcionalidad que representa en el interior peninsular la presencia de un enclave húmedo etc. convierten a este valle en un destino privilegiado para el turismo rural, que se desarrolla desde hace años, especialmente merced al impulso dado durante la ejecución de las iniciativas comunitarias Leader y Leader II, que han incluido a este subsector eritre sus objetivos prioritarios.

En un contexto de desarrollo del turismo rural, la situación de los cascos urbanos tiene interés, tanto por ser proveedores de alojamiento como por su condición de decorado, que plasma un ideal de vida rural fácilmente comercializable en los mercados urbanos. 
El casco urbano de El Torno muestra unas condiciones de conservación de la vivienda tradicional aceptables, y un gran número de viviendas deshabitadas disponibles como consecuencia de la despoblación y de la tendencia a la construcción de la vivienda nueva en áreas de nueva urbanización.

Por lo que respecta al plano, la planta viene condicionada por las sucesivas particiones por herencia de los solares, que da lugar a disposiciones poco funcionales, por lo que son abandonadas cuando es posible construir una nueva en la periferia, pero que sin embargo, resultan muy estéticas, constituyéndose en un recurso turístico más.

Junto a ellas, aparecen solares sin edificar, utilizados unos como huertos, abandonados otros, vestigios de diferentes etapas de crecimiento que han "capturado" en su interior antiguas tierras de labor. Actualmente se encuentran dispersos a modo de jardines privados, y son los únicos espacios abiertos de cierta entidad en un plano de calles estrechas y tortuosas, de perspectivas cortas. Muchos nunca serán edificados - dadas las tendencias de crecimiento que se observan-y suponen un recurso estético susceptible de ser aprovechado.

El principal de los problemas a la hora de aprovechar el viejo caserío para su explotación turística, estriba en las condiciones socioeconómicas en que se ha desarrollado la historia de El Torno, que son las de un pueblo pobre, de montaña, en el que la economía ha sido hasta hace pocas décadas subsistencial y precaria.

Esta pobreza, se ha traducido en el desarrollo de técnicas constructivas humildes: entre los materiales predominan el adobe y la piedra basta sobre el sillar, y la estructura de madera de roble sobre la de castaño, originando una construcción de mala calidad, que frecuentemente presenta un lamentable estado de conservación, con todas las patologías que suelen asociarse a este tipo de edificios.

A ello se une la dificultad de restauración, derivada de la falta de estabilidad de las fachadas, que difícilmente pueden ser consolidadas una vez que se retira la estructura original, en la que se integran como soporte.

Como consecuencia, en el cómputo de existencias, que podría abarcar a cerca del $60 \%$ del caserío, en la actualidad deshabitado, hay que descontar alrededor de un $70 \%$ de los edificios, cuya reconstrucción resulta prácticamente imposible o extremadamente costosa. Aún así, éstas conviven con otras muchas bien conservadas, que con seguridad son suficientes para cubrir la demanda que pueda producirse.

El segundo problema, de más difícil solución, es el deterioro del paisaje urbano que produce la construcción, especialmente durante la segunda 
mitad de nuestro siglo, de viviendas nuevas a lo largo de la carretera que une a El Torno con la N-110, y que se prolonga en parte del camino rural afirmado hacia la localidad de El Rebollar.

Éstas constituyen una pantalla que oculta el paisaje urbano tradicional, convirtiéndose en un elemento discordante que domina las perspectivas desde los accesos al pueblo, con una tipología constructiva estandarizada, en la que predomina la reducción de costes sobre cualquier criterio estético, que ha dado lugar a una construcción de escasa calidad, en alturas excesivas ${ }^{21}$ y $\sin$ criterio alguno de integración con el entorno.

A esta situación se ha llegado desde la apatía - cuando no connivencia- administrativa, prescindiéndose hasta los años 80 de ordenación urbanística, para pasarse a continuación a la redacción de unas normas subsidiarias de planeamiento de ámbito comarcal que, pese a su calidad, nunca llegaron a ser aplicadas. Los intentos de control del caos se han producido en la última década desde la Mancomunidad de Municipios, a través de su oficina de gestión urbanística, pero su gestión ha sido por lo menos ignorada por las autoridades municipales, cuando no abiertamente obstaculizada con criterios más electoralistas que de gestión.

Como corresponde a un espacio en el que la propiedad del suelo se encuentra dispersa y la explotación agrícola muy atomizada, en el paisaje de El Torno son muy abundantes los edificios destinados al servicio de ésta ${ }^{22}$.

Al igual que sucedia con las construcciones en el casco urbano, entre éstas podemos encontrar el contraste entre las más antiguas, con tipologías basadas en el uso de la piedra en mampostería seca (ocasionalmente prendida con argamasa) y cubierta de teja o piedra, y las modernas, en las que el material es el ladrillo, o bloque de hormigón, con frecuencia sin enfoscar, cuya cubierta suele levantarse con placas de uralita o chapa galvanizada ondulada.

En el caso de El Torno, con una antigua tradición ganadera extensiva, se pueden encontrar todavía vestigios de formas constructivas muy antiguas, que constituyen un valor más entre sus atractivos turísticos. Nos referimos fundamentalmente a pequeñas edificaciones de planta circular, levantadas en piedra basta, cuyos intersticios se rellenan de barro, con

21 Hay que mencionar aquí que el precio del suelo en el Valle del Jerte, especialmente en los cascos urbanos y sus zonas próximas es desorbitado.

${ }^{22}$ Tenados, o tenadas, aunque el uso de la forma "tinao" se encuentra generalizado tanto en el lenguaje oral como en el escrito. 
dinteles tallados desproporcionados a sus dimensiones en su única abertura al exterior, y cubiertos por falsas bóvedas de lajas muy interesantes.

De todo lo visto podemos concluir que nos encontramos ante un paisaje, muy antropizado que, pese a los problemas derivados de una concepción equivocada del desarrollo funcional, se presenta como un recurso de primera magnitud, cuya puesta en valor pasa por la recuperación de parte de lo perdido, la reutilización de lo que todavía existe, y la asunción de su rentabilidad por la población, que es no sólo su depositaria sino la beneficiaria directa de la conservación.

Naturalmente, a diferencia de lo que sucede en lugares orientados a formas de turismo basadas en la explotación de otro tipo de recursos (sol y playa), aquí no sería lógico plantearse un desarrollo económico centrado en el turismo, pero la explotación de los recursos paisajísticos puede constituir una fuente de ingresos complementarios y un yacimiento de empleo para cuya exposición es preciso lograr la pervivencia de las estructuras actuales del paisaje.

\section{DOTACIONES, SERVICIOS E INFRAESTRUCTURAS}

El Torno, pese a que apenas concentra una población de 970 habitantes, cuenta con una importante dotación pública, cuyos niveles de utilización actual se encuentran muy por debajo de los umbrales de saturación y ofrecen servicios de calidad más que aceptable: hogar del pensionista, edificio multiusos (consultorio médico, casa de cultura, biblioteca), escuela, instalaciones deportivas (cancha de usos múltiples - tenis, baloncesto-, campo de fútbol, piscina).

Por otra parte, por lo que respecta a la provisión de servicios por parte de la Administración Pública, tampoco son insuficientes: consulta médica diaria, recogida de basuras, asistencia a domicilio ${ }^{23}$, correos, diversos servicios proporcionados desde el Ayuntamiento (tramitación de solicitudes, asesoramiento, etc.), asistencia social (proporcionada por el Servicio Social de Base de la Mancomunidad de Municipios), ATRIA, diversos servicios ofrecidos por la Cooperativa ${ }^{24}$ (asesoramiento en agricultura, venta de insumos para la producción agrícola etc.).

\footnotetext{
23 Servicio cubierto por dos trabajadoras, una de las cuales es contratada por el Ayuntamiento (único caso en la comarca en que esto sucede), y otra por la Mancomunidad de Municipios.

${ }_{24}$ Sobre la condición de organismo público o privado de la cooperativa que discute más adelante.
} 
En resumen, puede considerarse que la acción pública, en cuanto respecta a la provisión de servicios, es más que suficiente, encontrándose, merced a la colaboración entre sus distintos estamentos, muy por encima de lo que podría esperarse.

Por lo que respecta a la dotación de servicios por parte del sector privado, podemos encontrar un comercio muy desarrollado en el subsector alimentación, con un total de 5 establecimientos (un ratio cercano a los 200 hab/establecimiento).

El subsector instituciones de crédito se encuentra representado por tres sucursales bancarias semipúblicas, echándose en falta la presencia de la banca privada, que concede mayor importancia a los criterios de rentabilidad que las cajas de ahorros a la hora de decidir los emplazamientos de sus oficinas.

Mención aparte merece el subsector hostelero, en el que encontramos, por una parte, dos casas rurales ( $y$ una más en construcción), junto a 7 bares, cafeterías y discotecas (ratio 139).

Junto a ellos, farmacia, venta minorista de muebles y electrodomésticos, peluquería, taxis (2), otros comercios minoristas (3), dos empresas dedicadas (aunque no de modo exclusivo) al transporte de mercancías, un almacén de piensos y fertilizantes, y dos de materiales de construcción (uno de ellos a $8 \mathrm{~km}$. del casco urbano, sobre la $\mathrm{N}-110$, orientado más al servicio comarcal que al local) completan un terciario bastante desarrollado.

No obstante, el desarrollo de Plasencia como centro comarcal de servicios, con la aparición de una gran superficie comercial, dos grandes supermercados, un multicine con siete salas, su comercio tradicional, una animada vida cultural, con conciertos, teatro, etc., y numerosos establecimientos comerciales y dedicados a los servicios a las personas, hace pensar en un incierto futuro para el comercio local, especialmente a partir del momento en que, concluidas las obras en marcha para el cambio de trazado, ensanchado y mejora del firme en la $\mathrm{N}-110$, se acorten los tiempos de desplazamiento hacia este centro comercial.

Sus posibilidades de supervivencia se encuentran condicionadas a la pluriactividad de los miembros de la unidad familiar, de tal modo que, en la mayor parte de los casos, la tienda es gestionada por uno de los miembros de la familia y constituye un complemento a los ingresos producidos por la explotación agraria, fenómeno que se reproduce en el resto de las actividades no agrarias en mayor o menor medida. 
Al contrario de lo dicho para las dotaciones, El Torno muestra una gran cantidad de carencias en su sistema de infraestructuras, que se manifiesta en una sensación de aislamiento, agudizada por su posición marginal respecto a la $\mathrm{N}-110$, de la que se encuentra a $6 \mathrm{~km}$, salvados por una carretera comarcal de montaña, en condiciones deficientes de conservación.

Por lo demás, apenas otras dos vías de comunicación parten del pueblo, caminos afirmados muy deficientes, en dirección a Cabezabellosa y El Rebollar.

La red de caminos rurales es también muy deficitaria, y su desarrollo se ve dificultado por consideraciones medioambientales, condicionándose de este modo la rentabilidad de la explotación agraria, al imponer importantes desembolsos en la adquisición de vehículos todo-terreno, o limitar en mucho la vida útil de los vehículos menos adecuados, pero asequibles, cuyo uso está generalizado en la comarca ${ }^{25}$. Pese a ello, durante los últimos años se han venido afirmando algunos de los caminos más utilizados para el servicio a las explotaciones en el término municipal, y el progreso de esta práctica será muy difícil de evitar.

Por lo que respecta a la infraestructura de telecomunicaciones, El Torno cuenta con acceso a la red telefónica conmutada y apenas una compañía ofrece servicio de telefonía móvil. El acceso a Internet es lento y de mala calidad por no existir nodo local accesible, lo que obliga a acceder a través de nodos genéricos generalmente colapsados; no existe acceso RDSI ni ADSL.

La red de agua potable podría ser mejorada, pues en la actualidad la depuración se realiza tan solo por medios químicos.

La evacuación de aguas residuales se ha venido realizando hasta ahora sin depurar sobre el Arroyo del Collado, cuyo caudal es incapaz de asimilarlos, pese a existir una vieja depuradora que nunca llegó a ser funcional por falta de presupuesto municipal, y que en la actualidad se encuentra muy deteriorada.

A lo largo de los próximos años se desarrollará en la Comarca un plan de depuración de aguas a cargo de la Diputación Provincial, encargándose su gestión a la Mancomunidad de Municipios, con lo que es de suponer que será resuelto lo que hasta ahora ha resultado un grave problema medioambiental.

\footnotetext{
25 Furgonetas tipo "combi", fundamentalmente, de las que existen 200 que, junto con los 19
} Todo-Terreno. 


\section{LA INTEGRACIÓN EN EL SISTEMA}

El Valle del Jerte se conforma en la actualidad como un espacio muy bien articulado, cuya organización descansa sobre, fundamentalmente, dos instituciones:

- En el plano económico, la Agrupación de Cooperativas del Valle del Jerte integra la comercialización de la producción y provee de servicios a las diversas cooperativas locales.

- En el político, la Mancomunidad de Municipios actúa como proveedora de servicios y constituye una primera escala de organización política territorial.

A partir de ambas, se genera desde tiempos muy tempranos una estructura integrada y una práctica de cooperación a escala comarcal, que resulta ejemplar en muchos aspectos, pese a mostrar disfuncionalidades que no son óbice para que nos encontremos ante un espacio en el que la integración comarcal se manifiesta a través de numerosos ejemplos.

Esta tradición de colaboración intermunicipal, se traduce en una cierta facilidad para la generación de esquemas de colaboración, que permiten el desarrollo de escalas suficientes como para abordar empresas ambiciosas. Así, surgen organizaciones como:

- La Sociedad para la Promoción y el Desarrollo del Valle del Jerte (SOPRODEVAJE): fórmula de colaboración entre el sector privado, el poder local y diversas organizaciones de participación ciudadana, que permiten, por ejemplo, la gestión de las Iniciativas Comunitarias Leader.

- El Consejo Regulador de la Denominación de Origen se encuentra actualmente en fase de consolidación, y puede ser uno de los factores de valorización del producto en el mercado nacional y europeo.

- Diferentes iniciativas de participación ciudadana, algunas de ellas muy interesantes, como la Asociación Comarcal de Mujeres del Valle del Jerte.

- Asociación de Turismo Rural.

Nos encontramos, por consiguiente, con una organización comarcal articulada que constituye un valor de primer orden para el desarrollo local, siempre y cuando se logre vencer la tentación del localismo, y se aprovechen efectivamente sus potencialidades. 
La Mancomunidad de Municipios del Valle del Jerte constituye una organización orientada a la prestación de servicios, entre cuyas funciones destacan la gestión de la recogida de basuras, el Servicio Social de Base, y la Oficina de Gestión Urbanística.

Sin embargo, y pese a esta orientación prioritaria, el paso del tiempo la ha llevado a constituirse en un escalón del poder territorial en la comarca de indudable trascendencia, donde se dirimen buena parte de las batallas locales y se coordina el trabajo de los poderes municipales.

En su seno se reproducen las tensiones del debate político, que llegan en ocasiones a bloquear su funcionamiento, pero pese a ello y a la excesiva tendencia al localismo, constituye una organización muy consolidada, que hay que valorar como un recurso a utilizar en el desarrollo comarcal.

La Agrupación de Cooperativas del Valle del Jerte es la cooperativa de $2 .^{\circ}$ grado, que constituye desde principios de los años 70 el corazón de la integración del valle del Jerte en el sistema económico, y uno de los emblemas del cooperativismo español.

El grueso de la producción frutícola de la comarca es comercializada desde aquí, convirtiéndola en la empresa que domina el mercado de la cereza a escala nacional, con un porcentaje variable de la producción que puede oscilar entre el $20 \%$ y el $35 \%$ del total, incrementándose cuando la comparación se limita al producto de calidad.

A sus funciones de comercialización une la de prestación de servicios, que van desde la investigación, hasta el desarrollo comarcal, mediante, por ejemplo, su participación en SOPRODEVAJE.

Al trabajo de la Agrupación puede achacarse la responsabilidad del mantenimiento del Valle del Jerte en el mercado (pese al continuo incremento de la superficie dedicada al cultivo del cerezo en áreas con menores costes de producción). Ello está siendo posible gracias al continuo trabajo de investigación, a las mejoras en las técnicas de conservación (hidrocooling), a la prospección permanente de nuevos mercados a escala continental, y al desarrollo de nuevos productos que están permitiendo formas de diversificación muy interesantes.

Todo ello se completa con una estrategia comercial basada en el diseño de una imagen homogénea de calidad, que afecta a la totalidad del entorno (turismo, etc.), y en la que trabajan en estrecha colaboración SOPRODEVAJE, FIFES, Denominación de Origen, etc., logrando la consecución de una posición de ventaja en el mercado, basada en los principios de diferenciación del producto, que permite mantenerse pese a ofrecer precios superiores a la media, merced a un estricto control de calidad. 
A su trabajo en los aspectos comerciales y servicios a los productores, la Agrupación suma el papel de aglutinadora del esfuerzo colectivo por el desarrollo del Valle del Jerte, habiéndose constituido como uno de los mecanismos de participación de la población en el desarrollo comarcal más importante. No en vano, aglutina en la actualidad a más de 4000 socios que, junto con sus familias, suponen la práctica totalidad de la población del Valle del Jerte, lo que la convierte en la primera institución comarcal.

SOPRODEVAJE constituye una empresa dedicada a la gestión del desarrollo comarcal, un Grupo de Acción Local, que permite la coordinación de los esfuerzos de instituciones públicas, financieras, empresariales, y organizaciones ciudadanas.

Entre sus logros destaca la gestión de las iniciativas comunitarias Leader y Adapt, y la coordinación de una estrategia de desarrollo, que constituye el único proyecto articulado de desarrollo a escala comarcal.

El Valle del Jerte, por tanto, constituye un sistema dotado de una red de interrelaciones y cooperación a escala comarcal que supone un activo de primer orden a la hora de abordar el desarrollo local de EI Torno.

De hecho, el éxito de cualquier iniciativa encaminada a este fin pasa necesariamente por conseguir un nivel óptimo de utilización del enorme recurso que supone esta estructura organizativa, que dota a la comarca de una herramienta muy potente de desarrollo.

\section{ACCIÓN INSTITUCIONAL Y ESTRATEGIAS COMARCALES}

En el marco de la integración en la Unión Europea, que amplía nuestro espacio de referencia y de toma de decisiones, en el del Estado de las Autonomías, que a su vez traslada la gestión a niveles territoriales del poder más próximos a la escala de los problemas, y en la tendencia actual al fomento de «lo local» en el desarrollo, se produce una alteración radical no sólo de las relaciones de poder, sino del mismo espíritu de las estrategias a emprender, que se traduce en diseños que aún se encuentran en fase de desarrollo.

Por lo que respecta al caso que nos ocupa, vamos a realizar un somero inventario de las estrategias en marcha más importantes, fijándonos especialmente en las encaminadas a:

1. Mejora de las bases técnicas de la explotación agraria y esquemas de comercialización del producto. 
2. Medidas de choque tendentes a la fijación de población en el medio rural extremeño.

3. Plasmación de las diferentes estrategias comunitarias de desarrollo.

En el Valle del Jerte resulta difícil situar el papel de la Agrupación de Cooperativas y sus cooperativas asociadas, puesto que, si bien nominalmente se trata de sociedades de derecho privado, en la práctica, debido al enorme número de socios, a su funcionamiento democrático, y a su importante capacidad de articulación social, se comporta de un modo que podríamos calificar de "pseudoinstitucional".

Este papel se refuerza por el hecho de constituir el principal interlocutor de la Administración en materia agrícola, y el socio comarcal en la mayor parte de las iniciativas, con lo que su papel bien puede asimilarse, salvando las diferencias en los modos de gestión, al de los organismos públicos.

El grueso de la acción institucional sobre la agricultura se organiza en torno a tres líneas de acción principales:

1. La mejora de las bases técnicas.

2. La captación de parte de las plusvalías generadas por el producto.

3. El desarrollo de nuevos canales de comercialización y métodos de preparación del producto que permitan incrementar el área de mercado.

En todos ellos aparece la Agrupación de Cooperativas, como promotor principal o como colaborador de la Administración.

Por lo que respecta a la primera destaca, tras el papel predominante de la Agrupación, el de la Agencia de Extensión Agraria, primer eslabón de contacto de la Administración con las estructuras agrarias comarcales.

En este mismo sentido, actúan las ATRIAs (Agrupación de Tratamientos Integrados en la Agricultura), cuyo papel consiste en el seguimiento detallado de una serie de parcelas piloto (pertenecientes y explotadas por agricultores locales), cuya evolución es objeto de una vigilancia permanente, decisiva especialmente para el desarrollo de métodos de control de plagas, problema este que podría llegar a ser importantísimo en un espacio en el que existe un monocultivo sobre más de $7000 \mathrm{Ha}$, que forman dos fajas prácticamente ininterrumpidas.

En cuanto a la fijación de plusvalías en la comarca, las acciones son muy variadas y se producen a todas las escalas: desde la gran destileria de aguardientes, o la almazara, gestionadas directamente por la Agrupa- 
ción, hasta las pequeñas industrias familiares dedicadas a la transformación de la producción primaria (mermeladas, dulces, quesos, embutidos etc.). En todos los casos, hay que destacar los altos niveles de calidad, que permiten la integración de las producciones en el objetivo de lograr una imagen comarcal en la que ésta es prioritaria.

Este aspecto, la calidad, es trascendental en el diseño estratégico, puesto que los costes de producción son más elevados que en el resto de las áreas productoras de cerezas en España, por lo que la competencia en precios resulta imposible. La supervivencia del cultivo sólo es posible merced al mantenimiento de unos niveles que posibiliten competir rentabilizando una imagen de marca que permita mantener precios elevados sin perder área de mercado, en base a la diferenciación del producto; esto es: la cereza es un producto, la cereza del Valle del Jerte es otro diferente.

Las estrategias de comercialización, por su parte, han experimentado a lo largo de las últimas décadas una ampliación de objetivos, y están encaminadas a la consolidación y ampliación de la presencia de la cereza en los mercados europeos, en los que se introduce merced a la calidad y a la imagen de producto artesanal de primor.

A tal fin se ha actuado desde diferentes enfoques, entre los que podemos destacar:

1. Estrategias encaminadas a popularizar el producto y conseguir una imagen de gran calidad.

2. Estrategias encaminadas a mejorar la calidad objetiva.

3. Estrategias encaminadas a mejorar las condiciones de conservación y transporte.

En este último aspecto, destaca la puesta en funcionamiento de una gran planta de procesado de la cereza que integra calibradoras, hidrocoolers (sistema de enfriado), envasadoras, paletizadoras, etc. y que logrará en próximas campañas, una vez solventados los problemas técnicos derivados de la integración en una única línea de producción de numerosas máquinas diferentes, la puesta en el mercado del producto en condiciones únicas en Europa ${ }^{26}$.

26 Ayuso, J. M. ${ }^{a}$, afirma, en el $n .^{\circ}$ correspondiente a marzo de 2000 de "La Picota" (periódico comarcal editado por F(FES): «Por así decirlo seremos la Coca-Cola entre los refrescos de cola, o el Danone entre los yogures", en una frase que sintetiza a la perfección el espiritu de la estrategia. 
Por lo que respecta a las medidas encaminadas a fijar población rural, su esencia es el AEPSA (antiguo PER), que si bien nominalmente estaba orientado a la mejora de las condiciones de vida de los trabajadores agrícolas por cuenta ajena, en la práctica ha servido fundamentalmente para cumplir el objetivo mencionado.

Naturalmente no es intención de este trabajo analizar las múltiples connotaciones que el Plan de Empleo Rural ha tendido en Extremadura, pero no es posible comprender la situación actual de ningún sector del medio rural extremeño sin su toma en consideración.

EI PER se ha manifestado como una excelente herramienta a la hora de solventar uno de los problemas estructurales más importantes con los que tenía que enfrentarse la Extremadura de los años 80: la existencia de un contingente de población rural con un déficit formativo importantísimo, cuyas bases económicas se estaban agotando, y al que no se le presentaba otra alternativa que el abandono del campo y la emigración a un sistema urbano que, inmerso en la crisis, era incapaz de acogerlo.

Mención aparte merece la inserción de las estrategias comarcales de desarrollo en las comunitarias: ya hemos mencionado la importancia de la aplicación de las diferentes iniciativas comunitarias a la comarca del Valle del Jerte, y en buena medida su incidencia, aunque haya sido de modo genérico, por lo que nos vamos a limitar ahora a sistematizar el enunciado de las más recientes y poner de manifiesto el modo en que la comarca ha trabajado para integrarse en el proceso de desarrollo rural impulsado desde la UE.

Naturalmente, éstas se han desarrollado en todos los casos con la participación de un socio nacional, la implicación de la Administración Pública Nacional y, generalmente, la gestión por parte de una entidad local o comarcal: 
Resumen de iniciativas comunitarias recientes en el Valle del Jerte

\begin{tabular}{|c|c|c|c|c|}
\hline Iniciativa & Periodo & Gestión & Promoción & Medidas \\
\hline Leader & $1996-2000$ & $\begin{array}{l}\text { SOPRO- } \\
\text { DEVAJE }\end{array}$ & Ídem & $\begin{array}{l}\text { B1. Apoyo técnico al desarrollo rural. } \\
\text { B2. Formación y ayudas al empleo. } \\
\text { B3. Turismo rural. } \\
\text { B4. Pymes, artesania y servicios. } \\
\text { B5. Valorización y comercialización agraria. } \\
\text { B6. Conservación y mejora del medio } \\
\text { ambiente. }\end{array}$ \\
\hline $\begin{array}{l}\text { Adapt } \\
\text { (Informa } \\
X X I \text { ) }\end{array}$ & $1998-2000$ & $\begin{array}{l}\text { SOPRO- } \\
\text { DEVAJE }\end{array}$ & İdem & $\begin{array}{l}\text { 1. Acciones de formación orientadas a trabaja- } \\
\text { dores con amenaza inminente de desempleo } \\
\text { 2. Anticipación, promoción de redes y nuevas } \\
\text { oportunidades de empleo. } \\
\text { 3. Adaptación de las estructuras y sistemas de } \\
\text { apoyo. } \\
\text { 4. Acciones de información, difusión y sensibi- } \\
\text { lización. }\end{array}$ \\
\hline $\begin{array}{l}\text { Now III } \\
\text { (Row) }\end{array}$ & $1998-2000$ & $\begin{array}{l}\text { Agrupación } \\
\text { de Coope- } \\
\text { rativas del } \\
\text { Valle del } \\
\text { Jerte }\end{array}$ & $\begin{array}{l}\text { Asociación } \\
\text { de anima- } \\
\text { ción y for- } \\
\text { mación } \\
\text { "Xerit" }\end{array}$ & $\begin{array}{l}\text { Eje1. Orientación y asesoramiento de la mujer. } \\
\text { Eje2. Acción positiva en las cooperativas. } \\
\text { Eje3. Actuaciones dirigidas a la cadena infor- } \\
\text { mática/telemática. } \\
\text { Eje4. Mujer e innovación tecnológica en la ca- } \\
\text { dena alimentaria. } \\
\text { Eje5. Creación de empresa: cadena vida pro- } \\
\text { tesional/personal. }\end{array}$ \\
\hline $\begin{array}{l}\text { Youthstart } \\
\text { II (NAY) }\end{array}$ & $1998-2000$ & $\begin{array}{l}\text { Mancomu- } \\
\text { nidad de } \\
\text { municipios } \\
\text { del Valle } \\
\text { del Jerte }\end{array}$ & Varios & $\begin{array}{l}\text { 1. Desarrollo de sistemas adecuados de forma- } \\
\text { ción, orientación, asesoramiento y empleo. } \\
\text { 2. Ofertas de planes de formación y de colo- } \\
\text { cación. } \\
\text { 3. Creación de puestos de trabajo. } \\
\text { 4. Divulgación de la información y acciones de } \\
\text { sensibilización. }\end{array}$ \\
\hline
\end{tabular}

Elaboración propia a partir de datos facilitados por SOPRODEVAJE.

Como podemos comprobar, el volumen de actuaciones y medidas es excesivo para ser analizado aquí en profundidad, pero ello no nos impide percibir que el grueso de las actuaciones inciden sobre el tejido empresarial - tratando de incentivar su modernización y diversificación- la cualificación de la mano de obra, para propiciar su adaptación a las nuevas exigencias del mercado de trabajo y nuevas necesidades del sistema territorial tal y como se pretende conformar, y la incorporación de colectivos con problemas al mercado laboral (mujeres y jóvenes).

A grandes rasgos, la materialización de las acciones se traduce en un importante esfuerzo encaminado a incidir sobre la formación de capital hu- 
mano en condiciones de responder a los nuevos requerimientos del sistema productivo (tanto en el sector agrario, como en lo secundario y terciario), al desarrollo de una potente red de cooperación interempresarial a escala comarcal y supracomarcal (junto con Valle de Ambroz, la Vera y Plasencia), y al fomento de la diversificación de fuentes de ingresos, en la que destaca el turismo rural, pero sin olvidar la industria agroalimentaria o la artesanía.

En conjunto, podemos afirmar que la comarca se muestra muy dinámi$\mathrm{ca}, \mathrm{y}$ ha generado una superestructura encaminada a promover su desarrollo utilizando todos los medios institucionales. Ello se ha visto favorecido por el extraordinario vigor del tejido social, articulado en torno a SOPRODEVAJE, que ha fomentado la dinamización de los sectores emprendedores de la sociedad jerteña y la captación de personal técnico muy especializado, propiciando la puesta en marcha de un proceso de formación permanente de agentes de desarrollo local que, a su vez, han movilizado a buena parte del tejido social.

En resumen, el proceso de integración del Valle del Jerte en las estrategias de desarrollo rural comunitario resulta ejemplar, y sus repercusiones se están traduciendo en un rápido desarrollo comarcal cuyos efectos probablemente serán mejor percibidos por la población en un futuro a medio plazo que en la actualidad.

\section{EL ESTADO DE LAS COSAS: POTENCIALIDADES Y BLOQUEOS}

Ya hemos podido comprobar que no son pocas las potencialidades que el pueblo cuenta en su haber: desde un medio natural que ofrece excelentes condiciones para la agricultura, la explotación turística etc., hasta una superestructura orientada al desarrollo comarcal muy importante, cuyas potencialidades parecen no estar siendo suficientemente utilizadas.

En principio, podemos afirmar que las potencialidades existen, y su explotación adecuada debe ser la base de cualquier estrategia de desarrollo. Sin embargo, parecen no ser suficientes para revertir, ni siquiera frenar, el proceso de despoblamiento desatado, de modo que habrá que estudiar los bloqueos para tratar de discernir cuáles son las causas.

Sin duda, el principal de ellos, y a la vez el más dificil de vencer, es el círculo vicioso de la despoblación del medio rural, con su capacidad para retroalimentarse. En el caso de El Torno, este proceso ha alcanzado ya una gran virulencia, y la "huida" del pueblo sigue manteniéndose como 
una alternativa que se contempla con agrado por una parte de los sectores más jóvenes de la población adulta.

Pero no es ese el único de los fenómenos de bloqueo que se producen, si no que podemos enunciar algunos más:

Esquema de potencialidades y bloqueos

\begin{tabular}{|c|c|c|}
\hline Factor & Potencialidades & Bloqueos \\
\hline Agricultura & $\begin{array}{l}\text { Clima muy favorable. } \\
\text { Infraestructura técnica. } \\
\text { Tradición. } \\
\text { Valor del producto. } \\
\text { Integración en el mercado. }\end{array}$ & $\begin{array}{l}\text { Insuficiencia de la superficie arable. } \\
\text { Dificultades de acceso a la explotación } \\
\text { por parte de los jóvenes. } \\
\text { Persistencia de prácticas y cultivos tradi- } \\
\text { cionales poco rentables. } \\
\text { Reparto desigual de las necesidades de } \\
\text { mano de obra a lo largo del año. }\end{array}$ \\
\hline Construcción & $\begin{array}{l}\text { Posibilidad de desarrollo del sector si } \\
\text { se afronta una estrategia de recupera- } \\
\text { ción del casco. }\end{array}$ & $\begin{array}{l}\text { Escasa especialización de la mano de } \\
\text { obra. } \\
\text { Falta de interés por la rehabilitación. }\end{array}$ \\
\hline Terciario & $\begin{array}{l}\text { Desarrollo del turismo rural. } \\
\text { Reserva de casas abandonadas. }\end{array}$ & $\begin{array}{l}\text { Problemas para competir del comercio } \\
\text { local. } \\
\text { Falta de iniciativa. } \\
\text { Descoordinación de la oferta. }\end{array}$ \\
\hline $\begin{array}{l}\text { Empleo en } \\
\text { general }\end{array}$ & $\begin{array}{l}\text { Tradición previa de pluriactividad } \\
\text { en la unidad familiar. } \\
\text { Contingente de población anciana. } \\
\text { Contingente de población adulta } \\
\text { susceptible de reciclaje profesional. }\end{array}$ & $\begin{array}{l}\text { Falta de empleo en sectores alternativos } \\
\text { a la agricultura. } \\
\text { Escasa cuantía de las pensiones de jubi- } \\
\text { lación de los agricultores autónomos. } \\
\text { Pérdida de la tradición artesanal. } \\
\text { Pérdida de buena parte de los sectores } \\
\text { dinámicos de la población. } \\
\text { Falta de cualificación en sectores alter- } \\
\text { nativos a la agricultura. }\end{array}$ \\
\hline Población & $\begin{array}{l}\text { Entorno de gran calidad. } \\
\text { Excelente imagen exterior. } \\
\text { Dotación de servicios y equipamientos. } \\
\text { Proximidad de un área urbana bien } \\
\text { dotada. }\end{array}$ & $\begin{array}{l}\text { Ambiente apático. } \\
\text { Falta de iniciativa. } \\
\text { Desequilibrios en la estructura demográ- } \\
\text { fica. }\end{array}$ \\
\hline
\end{tabular}

Fuente: Elaboración propia.

En síntesis, podemos deducir que el grueso de los frenos al desarrollo surgen de dos hechos principales: por una parte, la pérdida continuada de población ha desatado una dinámica difícil de revertir, fundamentalmente por lo que ha conllevado de desvitalización, con lo que la inercia tiende a reforzar el proceso hasta la extenuación demográfi- 
ca; por otra, las bases del sistema económico son disfuncionales: la agricultura no es capaz de proporcionar a la población un nivel de ingresos que haga la vida atractiva a la población externa, y no puede competir con las actividades de base urbana que están llamando continuamente a la población joven; el sector terciario se encuentra en retirada y sus funciones son asumidas por la cabecera comarcal, sobreviviendo sólo gracias a los bajos costes de la mano de obra familiar y a su función de fuente de ingresos complementaria a la agricultura; el secundario prácticamente no existe, si no es en relación con la construcción, en la que el trabajo no se remunera en igualdad de condiciones con el medio urbano, en parte por el predominio de las prácticas informales en el sector.

Por tanto, nos encontramos con que se manifiestan los problemas derivados del progreso técnico de nuestra sociedad sobre los espacios incapaces de adaptar sus estructuras a las nuevas exigencias del sistema productivo, y los niveles formativos de la población no permiten la incorporación a las ventajas de este progreso. La nueva economía destruye empleo en el sector primario y lo crea en determinados subsectores especializados del terciario, introduciendo un factor de segregación espacial, al primar a aquellos territorios cuya población dispone de niveles formativos e iniciativa que permitan la asunción de sus responsabilidades en el desarrollo. El Torno, con su población envejecida y la sangría permanente de los sectores más dinámicos y mejor formados, no se encuentra en la actualidad en condiciones de responder a estos desafíos por sus medios, y precisa de una fuerte y decidida intervención para situarse en una posición que le permita maximizar sus oportunidades.

\section{SINTESIS DE LA SITUACIÓN ACTUAL}

Hemos podido ver cómo nos encontramos ante un pueblo que, pese a encontrarse en una delicada situación de agotamiento demográfico, posee recursos suficientes como para poderse pensar que tiene oportunidades de desarrollo.

Por lo que respecta al mantenimiento de las bases económicas actuales es evidentemente necesario. El Torno dispone de un sistema de aprovechamiento agrícola del medio y unas estructuras suficientes como para poder considerar que la agricultura va a seguir constituyendo el elemento fundamental de su economía. Especializado en la producción de cerezas, localizado en un entorno capaz de aportarles valor, y pese a las 
amenazas de un mercado cada vez más competitivo, este cultivo parece capaz de mantenerse como el jefe de la economía local, máxime cuando a su alrededor se articulan las principales herramientas de integración comarcal.

Mención aparte merece el exceso de superficie dedicado al olivo, cuyos rendimientos se encuentran muy por debajo de los que ofrecen cerezo y castaño, por lo que quizás debiera relegarse tan sólo a las tierras cuyas características edáficas hagan inviable la explotación de aquellos, rompiendo la inercia que, por tradición, mantiene su cultivo, en un mercado que ha dejado de ser el tradicional (frente a una situación en la que el Valle del Jerte controla un porcentaje más que significativo de la producción nacional, e incluso europea, de cerezas de calidad, hasta el extremo de que el volumen de sus cosechas se traduce automáticamente en la coyuntura de precios de cada campaña; en el caso del olivo, especialmente cuando la producción se dedica al aceite, su inserción en un mercado de escala continental altamente especulativo y opaco) lo que hace irrazonable su mantenimiento en espacios agrarios capaces de producciones de mayor valor añadido.

En cualquier caso, no debemos olvidar que, pese a no contar con una explotación agraria capaz por sí sola de asegurar niveles suficientes de bienestar a los vecinos, su papel no es en absoluto desdeñable $y$, hasta la fecha, no ha aparecido ninguna fuente de ingresos que pueda considerarse sustitutoria. Frente a las voces que desde hace años han proclamado el final del papel agrario de los espacios rurales, la realidad se muestra terca y se empeña en demostrarnos que para la mayor parte de los espacios rurales no existe, por lo menos hasta ahora, un sistema de gestión del medio natural alternativo a la explotación agroganadera.

Por lo que respecta a la explotación del paisaje como recurso, mucho es lo que se ha hecho ya a escala comarcal, aunque la apuesta en El Torno no ha sido tan decidida como en otras localidades. En cualquier caso, conviene profundizar en su desarrollo, que debe - sin llegar a extremos que conduzcan a la destrucción minera del recurso turístico, provocando la degradación de sus valores medioambientales y paisajísticos por sobreexplotación- intensificar su aprovechamiento.

Muy problemática resulta la escasez de mano de obra previsible a medio plazo: en pocos años, los brazos serán insuficientes para garantizar la gestión de las actuales tierras de labor, y conviene diseñar una estrategia encaminada a importar y formar mano de obra capaz de afrontarla. La decisión de si ésta debe ser asalariada al servicio de los 
vecinos del pueblo, o si se debe incentivar el asentamiento de nuevos agricultores (para lo cual haría falta una fuerte acción institucional, dado el alto valor de los terrenos en la actualidad), es más política que técnica, pero en uno u otro sentido debe ser tomada sin demora, o el abandono de tierras llevará a una situación económica y medioambiental irreversible ${ }^{27}$.

El Torno cuenta, por lo demás, con un entorno natural privilegiado, que debe ser puesto en valor en todas sus vertientes, y su infrautilización constituye un derroche incomprensible: accesibilidad, belleza de sus paisajes, buen nivel de conservación de estos, alta capacidad de acogida de actividades turísticas, etc. todos ellos son factores muy favorables al desarrollo de un turismo de calidad que debe ser explotado. La búsqueda de soluciones imaginativas resulta a este respecto imprescindible para competir en un mercado en el que la oferta está creciendo de manera exponencial a lo largo de los últimos años, y cuya demanda, aún en progresión, tiene por fuerza que llegar a estabilizarse. Para su explotación se cuenta con una imagen previa de marca que debe aprovechar, y que hasta ahora ha venido utilizando un diseño de la oferta global en el que se combina calidad ambiental, calidad del producto, producción artesanal, propuestas de actividades, etc. ofreciendo una visión de la comarca muy atractiva, que constituye un recurso muy importante.

En definitiva, podemos afirmar que el medio es, salvo en los aspectos demográficos, muy favorable para el desarrollo económico de El Torno, que debería afrontar el diseño de una estrategia articulada e inserta en las comarcales, pero orientada, desde la particularidad de su problema concreto, a maximizar el valor de sus ventajas y dotarse de las condiciones que lo hagan atractivo para el asentamiento, de parte de su propia población emigrante, y quizás, nueva población que permita revitalizar las estructuras demográficas.

\section{BIBLIOGRAFÍA}

Barrientos Alfageme, G. (1990): "Geografía de Extremadura", Ed. Universitas. Badajoz.

Cevallos y Fernández de Córdoba, L., et alter (1979): “Árboles y Arbustos de la España Peninsular". ETS de Ingenieros de Montes. Madrid.

27 Es conocido el terrible problema de erosión que suele seguir al abandono de los campos aterrazados, que se derrumban originando procesos de acarcabamiento imparables, y que, dadas las características geomorfológicas del valle, llevaría a un afloramiento de la roca madre irreversible. 
COQUE, R. (1984): “Geomorfología». Alianza Editorial. Madrid.

Consejería de Economía, Industria y Comercio de la Junta de Extremadura (WEB), "Indicadores demográficos", http://www.juntaex.es.htm. Consulta a 22-8-2000.

Del CANTo Fresno, C., et alter (1992): "Desarrollo Rural: ejemplos europeos". IRYDA. Madrid.

- (1996): “Innovación y desarrollo en áreas rurales". MAPA. Madrid.

DerruAu, M. (1991): "Geomorfología». Ed. Ariel. Barcelona (2. ed.).

EDIHo (Web) "Agrupación de Cooperativas del Valle del Jerte", en "La Guía de las FrutasHortalizas de España» http://www.frutas-hortalizas.com/fic1491.htm. Consulta a 9-9-2000.

Gómez OreA, D. (1992): «Planificación Rural». Editorial Agrícola Española. Madrid.

- (1994): "Ordenación del territorio: una aproximación desde el medio físico". Editorial Agrícola Española/Instituto Tecnológico GeoMinero de España. Madrid.

INE (Web). "Base de datos" http://www.ine es.htm. Consulta a 20-8-2000.

JUNG, J. (1972): "La ordenación del espacio rural". Instituto de estudios de Administración Local. Madrid.

Junta de Extremadura: "Anuarios estadísticos de Extremadura (años 1989, 1992, 1995, 199798, 1999)». Ed. Junta de Extremadura. Badajoz, años citados en el título.

LEGuiNA, J. (1992): “Fundamentos de demografia". Ed. Siglo XXI. Paracuellos del Jarama, (Madrid).

MARTínez, C. y CAMACHO, G. (1980): “Marinaleda: huelga de hambre contra el hambre». Ed. Revolución. Madrid.

Ortega Hernández-Agero, C. et. alter (1989): "El libro rojo de los bosques españoles". ADENA-WWF España. Madrid.

Real Manzano, M. C. y SAnchez PÉrez, M. a (1998): “Acción positiva en las cooperativas". Inédito. Navaconcejo.

SAIM (1998): "La mujer y el mercado laboral en el Valle del Jerte". Inédito. Navaconcejo.

SALAYA MONSELL, R. (1997): “Extremadura". Inédito. Navaconcejo.

ToRrojA, J. A.: “Normas subsidiarias de planeamiento del Valle del Jerte (Memoria)". Inédito. Madrid.

VVAA (1985): “Historia Agraria de la España Contemporánea». Editorial Crítica. Barcelona.

VVAA (1994): "Iniciativa Comunitaria Youthstart II: New activities for youth (NAY), Memoria". Inédito. Valdastillas, 1994.

VVAA (1995): "Iniciativa Comunitaria NOW III: Rural Opotunities For Women (ROW), Memoria". Inédito. Valdastillas.

VVAA (1994): "Iniciativa Comunitaria ADAPT: Informa XXI. Memoria". Inédito. Valdastillas.

VVAA (2000): «Memoria de actuaciones realizadas a 31-12-1999. Iniciativa comunitaria Leader IIm. Inédito. Valdastillas, 2000.

VVAA (1995): “Normativa para la aplicación de la Iniciativa Comunitaria Leader II del Valle del Jerte". Inédito. Valdastillas. 